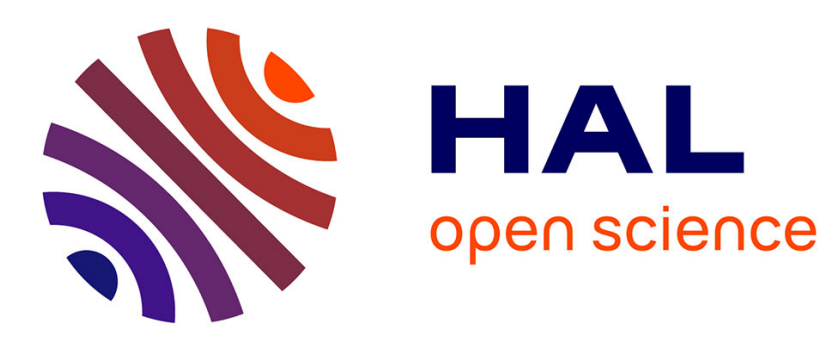

\title{
A Possible Minimum Relevance Requirement for a Statistical Approach in a Reverberation Chamber
}

Florian Monsef, Andrea Cozza

\section{To cite this version:}

Florian Monsef, Andrea Cozza. A Possible Minimum Relevance Requirement for a Statistical Approach in a Reverberation Chamber. IEEE Transactions on Electromagnetic Compatibility, 2015, 57 (6), pp.1728-1731. 10.1109/TEMC.2015.2464318 . hal-01241325

\section{HAL Id: hal-01241325}

\section{https://hal-centralesupelec.archives-ouvertes.fr/hal-01241325}

Submitted on 11 Dec 2015

HAL is a multi-disciplinary open access archive for the deposit and dissemination of scientific research documents, whether they are published or not. The documents may come from teaching and research institutions in France or abroad, or from public or private research centers.
L'archive ouverte pluridisciplinaire HAL, est destinée au dépôt et à la diffusion de documents scientifiques de niveau recherche, publiés ou non, émanant des établissements d'enseignement et de recherche français ou étrangers, des laboratoires publics ou privés. 


\title{
Letters
}

\section{A Possible Minimum Relevance Requirement for a Statistical Approach in a Reverberation Chamber}

\author{
Florian Monsef, Member, IEEE, and Andrea Cozza, Senior Member, IEEE
}

\begin{abstract}
Modal overlap in a reverberation chamber (RC) involves, strictly speaking, an infinite number of modes in the field statistics. The degree of overmodedness of an RC is often assessed by introducing an arbitrary reference bandwidth in which a number of overlapping modes is computed. We introduce a statistical model of the field in which the number of modes is rigorously finite. The model is chosen such that the number of degrees of freedom (DOF) of the field can be assessed. The number of DOF is assessed by considering the degree of homogeneity of the average power received by a linearly polarized antenna. It is considered that a statistical approach requires at least a single degree of freedom. Based on this criterion, the minimum frequency at which a statistical approach would make sense is evaluated and compared to the lowest useable frequency (LUF) commonly considered in EMC.
\end{abstract}

Index Terms-Cavity resonators, electromagnetic compatibility (EMC), modal analysis, parametric statistics, reverberation chamber (RC).

\section{INTRODUCTION}

I $\mathrm{N}$ order to deal with the complexity of the electromagnetic (EM) field inside reverberation chambers (RCs), a statistical approach is commonly used to estimate the degree of homogeneity of the mean and maximum power level that can be expected [1]-[3]. The power, referred to as $P$, is meant as being measured on a given rectangular field component by a linearly polarized antenna.

Due to wall losses and other loss mechanisms, the EM field results from the superposition of modes regarded as random normal modes. As soon as a certain number of modes is efficiently excited, the field can be assumed to follow a Gaussian law, a direct result from the central-limit theorem [4]. If the number of excited modes happened to raise, fluctuations of $P$ about its mean value, i.e., its relative variance (or variability), would remain steady. It corresponds to an overmoded regime for which an infinite number of modes is ideally and theoretically assumed. The relative variance of the measured mean power tends to unity, as $P$ follows a $\chi_{2}^{2}$ law. A recent study [5] has highlighted the rate at which power variability tends toward its asymptotic value.

Manuscript received June 29, 2015; accepted July 15, 2015.

The authors are at the GeePS Lab, UMR8507, Universite Paris-Sud, CentraleSupelec, CNRS, UPMC, 91192 Gif-sur-Yvette, France (e-mail: florian.monsef@u-psud.fr).

Color versions of one or more of the figures in this paper are available online at http://ieeexplore.ieee.org.

Digital Object Identifier 10.1109/TEMC.2015.2464318
Each contributing mode could be thought of acting as a degree of freedom (DOF). The larger the number of DOF, the more Gaussian the field will be. Although intuitive, the correspondence between the number of excited mode and the number of DOF is not straightforward for the following reason. The frequency response of the modes are of Lorentzian shape. This means that the number of overlapping modes is rigorously infinite. One could bound the number of overlapping modes by setting a bandwidth of reference. In [6] for instance, the $-3 \mathrm{~dB}$ modal bandwidth was chosen. This choice can be a metric that provides the degree of modal overlap, but does not provide the number of modes that contribute to a given level of field homogeneity [7] . In any case, whatever the chosen bandwidth, the modal overlap involves theoretically an infinite number of modes.

This study tackles the problem in another way. It proposes an equivalent statistical model that, on the one hand, makes not possible the modal overlap to be infinite, and on the other hand, provides the same degree of homogeneity in a chamber, i.e., leads to the expected power variability. It is essentially a way to circumvent the problem of the tails of the frequency response of the modes. As we will see, the bandwidth over which the modal overlap is regarded is however related to the choice of some model parameters. Interestingly, this proposed approach allows us to define properly the concept of DOF and highlights the results derived in [8]. Finally, the model allows us to propose a criterion that could be a possible answer to the pending question dealing with the frequency from which a statistical approach makes sense in an RC.

After defining what is meant by DOF in Section II, the equivalent statistical model of the electric field will be exposed. The assessment of the number of DOF will also be presented. Section III will focus on the validation of the proposed model by means of Monte Carlo (MC) simulation. Finally, the frequency from which a statistical approach makes sense in an $\mathrm{RC}$ will be presented in Section IV.

\section{Modal Overlap AND Degrees of FreEdom}

\section{A. Degrees of Freedom: Definition}

It is important to define what is meant by DOF in order to understand the interest of the approach presented herein. Let us consider a random variable $Y$ expressed as follows:

$$
Y=\sum_{i=1}^{N} X_{i}
$$


where $X_{i}$ are independent and identically distributed (i.i.d.) random variables. The number $N$ is defined as the number of DOF of $Y$.

A typical example of such definition is met with a $\chi_{N}^{2}$ variable where the $X_{i}$ are $\chi_{1}^{2}$ distributed.

The basic idea of the paper is to introduce a way to express the electric field on the model given by (1), the interest of it will become clearer thereafter.

\section{B. Matching a Physical Model to a Statistical Field Model}

The first step of the proposed approach consists in recalling the physics that describes the electric field in an RC. To that aim, a modal approach is used, and the electric field $\mathbf{E}$ at a position $\mathbf{r}$ and at a frequency $f$ can be expressed as follows [8], [9]:

$$
\mathbf{E}(\mathbf{r}, f)=\sum_{i=1}^{\infty} \gamma_{i} \psi_{i}(f) \mathbf{e}_{i}(\mathbf{r})
$$

where $\mathbf{e}_{i}$ is the eigenvector of the $i$ th mode, which is assumed not to have a specific form, $\psi_{i}$ is the frequency response of the $i$ th mode, and $\gamma_{i}$ is the modal weight, i.e., the coupling constant of the source to the $i$ th eigenmode.

Limiting the analysis to a single position $\mathbf{r}$, the following factorization for the modal topographies can be used:

$$
\mathbf{e}_{i}(\mathbf{r})=e_{i}(\mathbf{r}) \hat{\xi}_{i}(\mathbf{r})
$$

where $\hat{\xi}_{i}(\mathbf{r})$ is a unitary polarization vector assumed to be uniformly distributed over $4 \pi \mathrm{sr}$.

As explained in [9], it is convenient to regard the set $\left\{\gamma_{i} e_{i}(\mathbf{r})\right\}$ as a set of a single random variable $\tilde{\gamma}_{i}$ defined as

$$
\tilde{\gamma}_{i}=\gamma_{i} e_{i}(\mathbf{r})
$$

referred to as equivalent modal weights [9]. The real and imaginary parts of $\tilde{\gamma}_{i}$ are assumed to follow a centered normal law.

As done in [5], [8], and [9], we consider three sets of modal parameters, i.e., $\tilde{\gamma}_{i}, \psi_{i}$, and $\hat{\xi}_{i}$ as random variables. The main assumptions required are at two levels. First, the modal parameters of different sets are independent, second, the parameters within the same set are i.i.d.

Now, the electric field described by (2) corresponds to a sum of weighted random variables, the weight function being $\psi_{i}(f)$. If we want to highlight the number of DOF for the case of the electric field, a rectangular shaped weight function has to be considered; the weight function, referred to as $\psi_{i, e}(f)$, would be of width $B_{w}$ and of height $A_{e q} \forall i$.

By doing so, we are introducing fictitious modes that have rectangular shaped frequency response. So, these modes are, or totally excited, or not at all. When these are excited, their contribution is equal to their neighbors.

In other terms, we are introducing an arbitrary electric field model. Accordingly, an equivalent electric field, referred to as $\mathbf{E}_{\mathbf{e}}(\mathbf{r}, f)$ must be considered; it is still expressed as a modal expansion as follows:

$$
\mathbf{E}_{\mathbf{e}}(\mathbf{r}, f)=\sum_{i=1}^{\infty} \tilde{\gamma}_{i, e} \psi_{i, e}(f) \hat{\xi}_{i, e}(\mathbf{r})
$$

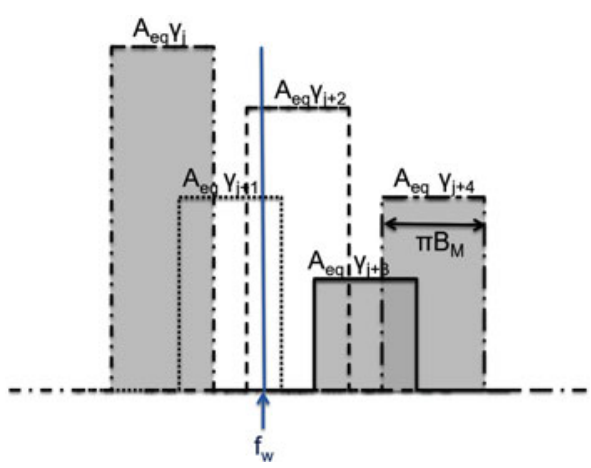

Fig. 1. Illustration of the overlap using the fictitious-modes model. Given the shape of their frequency response, only a finite number of those is excited - two in the illustrated case.

where $\tilde{\gamma}_{i, e}, \hat{\xi}_{i, e}(\mathbf{r})$ and $\psi_{i, e}(f)$ refer to equivalent modal quantities. However, these do not follow necessarily the same statistics as the modal quantities in (2) as they are related to fictitious modes.

The infinite modal-overlap issue at given frequency $f_{w}$ is then circumvented as shown in Fig. 1. The sketch highlights indeed that only a finite number of fictitious modes intervene in (5). It follows that $\mathbf{E}_{\mathbf{e}}(\mathbf{r}, f)$ can be simplified and reads

$$
\mathbf{E}_{\mathbf{e}}(\mathbf{r}, f)=\sum_{i=1}^{N} \tilde{\gamma}_{i, e} \hat{\xi}_{i, e}(\mathbf{r})
$$

where $N$ is the number of DOF, i.e., of fictitious modes, whose number will be specified in the next section. It is worth noting that the expression in (6) follows indeed the form given by (1).

Provided that the degree of homogeneity of the average power is the quantity of interest, its variability, referred to as $\varsigma_{P}^{2}$, must remain the same no matter what model is used. In other terms, (2) and (6) must lead to the same expression of the variability. This one was shown to be expressed in [5] as

$$
\varsigma_{P}^{2}=1+\frac{8}{5 \pi M_{M}}
$$

where $M_{M}$ stands for the average number of real modes overlapping in the $-3 \mathrm{~dB}$ modal bandwidth.

We chose $\tilde{\gamma}_{i, e}$ and $\hat{\xi}_{i, e}(\mathbf{r})$ to share $\tilde{\gamma}_{i}$ and $\hat{\xi}_{i}(\mathbf{r})$ properties, respectively. This choice is motivated by the fact that the main issue is only linked to the tails of the frequency responses. Accordingly, we do not have any reason to consider that $\tilde{\gamma}_{i, e}$ and $\hat{\xi}_{i, e}(\mathbf{r})$ follow different probability laws than $\tilde{\gamma}_{i}$ and $\hat{\xi}_{i}(\mathbf{r})$.

Now, if (6) is considered, by using the approach used in [5], it is easy to show that $\varsigma_{P}^{2}$ reads

$$
\varsigma_{P}^{2}=1+\frac{\Gamma-2}{N}
$$

where $\Gamma$ is defined as

$$
\Gamma=\frac{\mu_{4}}{\mu_{2}^{2}} \frac{\nu_{4}}{\nu_{2}^{2}} \frac{\kappa_{4}}{\kappa_{2}^{2}}
$$

From [5] and [9], $\mu_{n}=\mathrm{E}_{\tilde{\gamma}_{\mathrm{i}, \mathrm{e}}}\left[\left|\tilde{\gamma}_{i, e}\right|^{n}\right], \kappa_{n}=\mathrm{E}_{\mathrm{u}_{\mathrm{i}, \mathrm{e}}}\left[\left|u_{i, e}\right|^{n}\right]$, and $\nu_{n}=\mathrm{E}_{\psi}\left[\left|\psi_{i, e}(f)\right|^{n}\right]$, where $\mathrm{E}_{\mathrm{x}}[\cdot]$ is the ensemble average operator applied to $x$. 
Given that $\tilde{\gamma}_{i, e}$ and $u_{i, e}$ follow a Gaussian and a uniform law, ${ }^{1}$ respectively, we can show that $\Gamma$ reads

$$
\Gamma=\frac{18}{5} \frac{\nu_{4}}{\nu_{2}^{2}}
$$

Moreover, the frequency responses of the model being of rectangular shape, we can easily show that

$$
\begin{aligned}
& \nu_{2}=m\left(f_{w}\right) A_{e q}^{2} B_{w} \\
& \nu_{4}=m\left(f_{w}\right) A_{e q}^{4} B_{w}
\end{aligned}
$$

where $m\left(f_{w}\right)$ is the modal density in mode/ $\mathrm{Hz}$ at working frequency $f_{w}$. [5]

If we use the real-modes model, we have $\nu_{2}$ and $\nu_{4}$ such that

$$
\begin{aligned}
& \nu_{2}=\frac{\pi m\left(f_{w}\right)}{2 B_{M}} \\
& \nu_{4}=\frac{\pi m\left(f_{w}\right)}{4 B_{M}^{3}} .
\end{aligned}
$$

In order to match (11) to (12), the frequency responses of the fictitious modes must be chosen such that

$$
A_{e q}=\frac{1}{B_{M} \sqrt{2}}
$$

and

$$
B_{w}=\pi B_{M} .
$$

We can make a total analogy with the equivalent noise bandwidth, referred to as $B_{N}$, of a filter; $B_{N}$ is the bandwidth of an ideal filter (rectangular response) for which the power noise is the same than the one that we have with a real filter. This concept has been transposed herein such that the equivalent bandwidth provides the same statistical moments. This bandwidth may be referred to as the statistical bandwidth as proposed in [8].

If we had taken the electric energy density $W$ as quantity of interest, as done in [9] for instance, the related variability, referred to as $\varsigma_{W}^{2}$ would be the metric to consider. In order to match the two first moments of $W$, we can show that the conditions needed on $A_{e q}$ and $B_{w}$ remain unchanged.

It is worth stressing that other shapes (triangles, etc.) of frequency responses could have led to finite modal overlap, but those would still have behaved as weighting functions. In a strict sense, the bandwidth, over which modal overlap is estimated, depends on the shape of the frequency response. However, the assessment of the number of DOF would not be possible for nonrectangular shapes. So the coupling of the DOF constraint enforces a single bandwidth value, the one given by (14).

\section{Number of Degrees of Freedom}

Now that the bandwidth $B_{w}$ is identified as being $\pi$ times the modal bandwidth, the sketch of Fig. 1 allows us to assess easily the number of fictitious modes, or in other words, the number of DOF.

\footnotetext{
${ }^{1}$ See $[5$, Sec. III $]$.
}

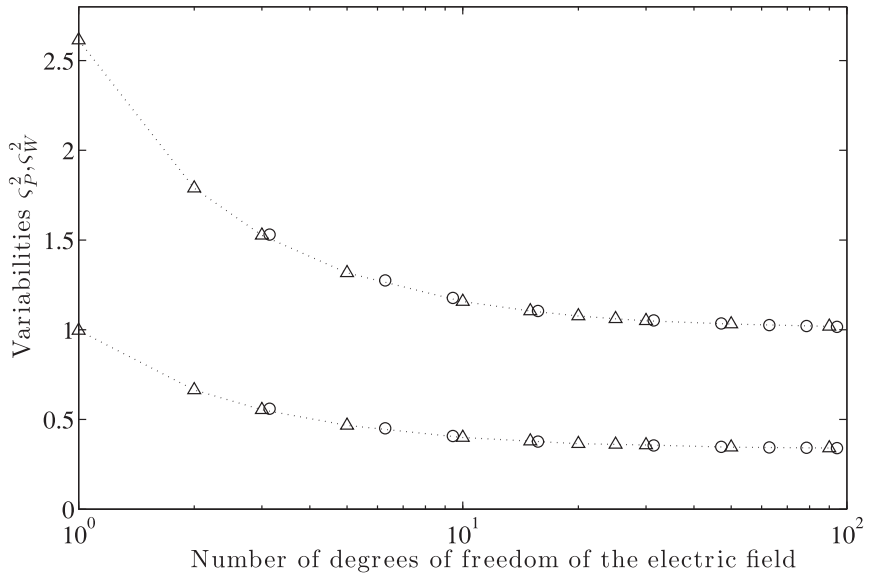

Fig. 2. Relative variances of the power measured along a given cartesian component (upper curve) and of the electric-energy density (lower curve) obtained by MC simulations using (2) (circles) and (6) (triangles). These are plotted as a function of the number of degrees of freedom of the field.

The average distance between continuous modes being $1 / m\left(f_{w}\right)$, the number $N$ of DOF is such that

$$
N=m\left(f_{w}\right) B_{w}=\pi M_{M} .
$$

This number corresponds to what was called the "effective number" of excited modes in [8]. The problem is tackled here from another angle, highlighting that these "modes" correspond in fact, from a statistical point of view, to a number of DOF.

We emphasize that the number $N$ must not be regarded as a number of real modes that contribute to the field statistics otherwise leading to an improper variability level as shown in [7]. This is especially striking at low modal overlap. For this case, the modes that contribute to the statistics are poorly excited due to their Lorentzian-shaped frequency response. This impose to consider real modes spreading over a wide frequency band (50 $B_{M}$ according to [7] for $M_{M}=1$ ); with the DOF model, the fictitious modes are entirely excited and the statistics does not need such a wide frequency band to converge.

\section{MOdel VALIDATION}

The form obtained in (6) can make one skeptical about the validity of such a simple equivalent model of the electric field. In order to raise doubts, we have computed, on the one hand, $\varsigma_{P}^{2}$ and $\varsigma_{W}^{2}$ using MC simulations for the following number of DOF: $1,2,3,5,10,15,20,25,30,50$, and 90 . On the other hand, we have computed MC simulations by using the realmodes model given by (2). Fig. 2 shows the resulting relative variances, i.e., those obtained using the physical model (circles) and the equivalent model (triangles), respectively. In this procedure, 5000 realizations and 500 independent stirrer states were considered.

When using physical modes, the computation of the moments of $P$ assumes that a sufficient number of (real) modes is considered in the simulation as explained in [7]. In a strict manner, the number of real modes included is always truncated. This does not happen with the equivalent model, since this one is defined as being composed of a finite number of DOF as expressed in 
(6). Besides, it is clear that the number of DOF taken into account in the simulations are integer values since the sum in (6) is discrete. It allows us not to run into the question of setting a sufficiently large bandwidth.

Relative variances obtained with the physical model on the one hand, and with the statistical model on the other hand, are found to be in good agreement. This allows us to validate the fact that the relative variance obtained using (6) matches the "real" one obtained by using classical modal theory.

\section{DOF AND RELEVANCE OF A STATISTICAL APPROACH}

The advantage of the statistical model lies in the simple overlap model that it provides. If a single DOF is excited would not it be a reasonable criterion to consider it as the minimum requirement to consider the relevance of a statistical approach? We propose to regard the frequency for which a single DOF is considered as the minimum frequency from which a statistical modeling of the field makes sense.

We emphasize to distinguish this frequency, referred to as $f_{m}$, from the lowest useable frequency (LUF). This one is indeed defined over a homogeneity criterion of the maximum stress [10]. The link between the variabilities considered herein and the maximum stress being not straightforward, $f_{m}$ and the LUF are clearly different quantities. Note however that $f_{m}$ is likely to be lower than the LUF.

In the light of the above, $f_{m}$ is such that

$$
\mathbf{E}_{\mathbf{e}}\left(\mathbf{r}, f_{m}\right) \triangleq \mathbf{E}_{\mathbf{e}}(\mathbf{r}, f), N=1 \text {. }
$$

In order to determine $f_{m}$, we must solve the following equation:

$$
N\left(f_{m}\right)=m\left(f_{m}\right) \pi B_{M}=1 .
$$

Recalling that $B_{M}=f_{m} / Q$, where $Q \equiv Q\left(f_{m}\right)$ is the composite quality factor [11], relation (17) can be recast and $f_{m}$ must satisfy

$$
m\left(f_{m}\right) \pi f_{m}=Q\left(f_{m}\right) .
$$

The frequency range under consideration corresponds to the case where the RC is undermoded. Accordingly, we prefer to adopt the following modal-density expression:

$$
m(f) \simeq \frac{8 \pi V f^{2}}{c_{0}^{3}}-\frac{a+b+c}{c_{0}}
$$

valid for the cuboid case, note that the second term is a correcting term for low frequencies [11] where $a, b$, and $c$ correspond to the cuboid edges.

We observed in [8] that the quality factor of the chamber was quite linear with the frequency. The study was performed for a minimum frequency of $700 \mathrm{MHz}$, i.e., for a frequency above the $550 \mathrm{MHz}$ LUF of the chamber. In order to have a more accurate insight on the value of $f_{m}$, we processed measurements between $200 \mathrm{MHz}$ and $1 \mathrm{GHz}$. Note that for these measurements, the RC was empty. Fig. 3 shows the evolution of the quality factor, the left-hand term of (18) is superimposed. The intersection point provides the solution for $f_{m}$ which turns out to be about 250 $\mathrm{MHz}$, i.e., well below the LUF as expected.

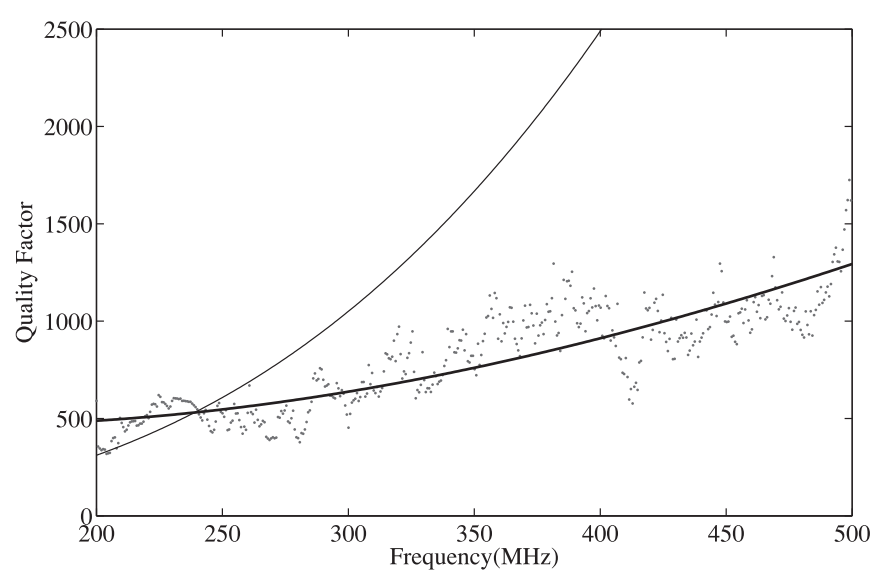

Fig. 3. Estimated $Q$ factor (symbols) with the average trend (bold line). $m(f) * \pi f$ (thin solid line) has been superposed. The intersection point highlights the frequency for which a single DOF is found.

\section{CONCLUSION}

A statistical model of the electric field has been presented. It is based on a square frequency response of the modes which allows us to highlight the number of DOF of the field. The interest of this choice lies in the fact that only a strictly finite number of modes overlap. The number of DOF is defined over a criterion based on the variability of the power measured along a linearly polarized antenna and/or of the electric energy density.

If less than a single DOF is considered, the relevance of a statistical approach is cut down. Accordingly the frequency at which a single DOF is excited was proposed to be regarded as the lower bound from which a statistical approach would make sense in a reverberation chamber. The frequency is expected to be smaller than the LUF commonly considered in EMC.

\section{REFERENCES}

[1] Reverberation Chamber Test Methods, IEC Standard 61000-4-21, 2003.

[2] T. H. Lehman, "A statistical theory of electromagnetic fields in complex cavities," Interaction Notes, USAF Phillips Laboratory, NM, USA, Note 494, May 1993.

[3] J. Kostas and B. Boverie, "Statistical model for a mode-stirred chamber," IEEE Trans. Electromagn. Compat., vol. 33, no. 4, pp. 366-370, Nov. 1991.

[4] A. Papoulis, Probability, Random Variables, and Stochastic Processes, 2nd ed. New York, NY, USA: McGraw-Hill, 1984, p. 168.

[5] F. Monsef and A. Cozza, "Variability and confidence intervals of the power measured in a reverberation chamber," IEEE Trans. Electromagn. Compat., vol. 56, no. 5, pp. 1238-1241, Oct. 2014.

[6] L. Arnaut and G. Gradoni, "Probability distribution of the quality factor of a mode-stirred reverberation chamber," IEEE Trans. Electromagn. Compat., vol. 55, no. 1, pp. 35-44, Feb. 2013.

[7] F. Monsef, "Why a reverberation chamber works at low modal overlap," IEEE Trans. Electromagn. Compat., vol. 54, no. 6, pp. 1314-1317, Dec. 2012.

[8] F. Monsef and A. Cozza, "Average number of significant modes excited in a mode-stirred reverberation chamber," IEEE Trans. Electromagn. Compat., vol. 56, no. 2, pp. 259-265, Apr. 2014.

[9] A. Cozza, "The role of losses in the definition of the overmoded condition for reverberation chambers and their statistics," IEEE Trans. Electromagn. Compat., vol. 53, no. 2, pp. 296-307, May 2011.

[10] Reverberation Chamber Test Methods, IEC Standard 61000-4-21, 2011.

[11] B. Liu, D. Chang, and M. Ma, "Eigenmodes and the composite quality factor of a reverberating chamber," U.S. Nat. Bur. Stand., Tech. Rep. 1066, 1983. 